\title{
THE ECCENTRIC CONNECTIVITY INDEX OF ARMCHAIR POLYHEX NANOTUBES
}

\author{
Mahboubeh Saheli, Ali Reza Ashrafi \\ Institute of Nanoscience and Nanotechnology, University of Kashan, \\ Kashan 87317-51167, Iran \\ Ashrafi@kashanu.ac.ir
}

\begin{abstract}
The eccentric connectivity index $\xi(G)$ of the graph $G$ is defined as $\xi(G)=\Sigma_{\mathrm{u} \in V(G)} \operatorname{deg}(u) \varepsilon(u)$ where $\operatorname{deg}(u)$ denotes the degree of vertex $u$ and $\varepsilon(u)$ is the largest distance between $u$ and any other vertex $v$ of $G$. In this paper an exact expression for the eccentric connectivity index of an armchair polyhex nanotube is given.
\end{abstract}

Key words: eccentric connectivity index; armchair nanotube

\section{ЕКСЦЕНТИРЧЕН ИНДЕКС НА ПОВРЗУВАЊЕ НА НАНОЦЕВКИ СО ФОРМА НА ПОЛИХЕКСАГОНАЛНА ФОТЕЛЈА}

\begin{abstract}
Ексцентричен индекс на поврзување $\xi(G)$ на графот $G$ е дефиниран како $\xi(G)=\Sigma_{\mathrm{u} \in V(G)} \operatorname{deg}(u) \varepsilon(u)$, каде $\operatorname{deg}(u)$ го означува степенот на темето $u$, а $\varepsilon(u)$ е најголемото растојание помеѓу $u$ и кое било друго теме $v$ од $G$. Во овој труд е изведен егзактен израз за ексцентричен индекс на поврзување на наноцевки со форма на полихексагонална фотелја.
\end{abstract}

Клучни зборови: ексцентричен индекс на поврзување; наноцевка со форма на фотелја

\section{INTRODUCTION}

The graph theory has successfully provided chemists with a variety of very useful tools, namely, the topological index. A topological index is a numeric quantity of the structural graph of a molecule. This graph has atoms as vertices and two atoms are adjacent if there is a bond between them.

Suppose $u$ and $v$ are vertices of the graph $G$. We define their distance $d(u, v)$ as the length of the shortest path connecting $u$ and $v$ in $G$. For a given vertex $u$ of $V(G)$ its eccentricity $\varepsilon(u)$ is the largest distance between $u$ and any other vertex $v$ of $G$. The maximum eccentricity over all vertices of $G$ is called the diameter of $G$ and denoted by $D(G)$ and the minimum eccentricity among the vertices of $G$ is called radius of $G$ and denoted by $R(G)$. The set of vertices whose eccentricity is equal to the radius of $G$ is called the center of $G$. It is well known that each tree has either one or two vertices in its cen- ter. The eccentric connectivity index $\xi(G)$ of the graph $G$ is defined as $\xi(G)=\sum_{u \in V(G)} \operatorname{deg}(u) \varepsilon(u)$ [1-3]. The mathematical properties of this topological index are studied in some recent papers [49].

In some research papers [10-16], the authors have computed some topological indices of armchair nanotubes. The aim of this article is to continue this problem and compute the eccentric connectivity index of an armchair polyhex nanotube, Figure 1.

\section{MAIN RESULTS}

In this section, the eccentric connectivity index of the molecular graph of an armchair polyhex nanotube $\operatorname{TUVC}_{6}[\mathrm{p}, \mathrm{q}]$ is computed, where $p$ is the number of rows in $2 \mathrm{D}$ perception of $\mathrm{TUVC}_{6}[\mathrm{p}, \mathrm{q}]$ (Figure 2), and $q$ is the number of vertical crenels. 
For simplicity, we denote this nanotube by $T=$ $T[p, q]$. Notice that $q$ is even. Set $E=E(T)$ and $V=$ $\mathrm{V}(\mathrm{T})$. Obviously, $|V|=p q$ and $|E|=(p-1) q+p q / 2$ $=(3 / 2 p-1) q$. To compute the eccentric connectivity index of this nanotube, two cases $p$ is odd and even are considered.

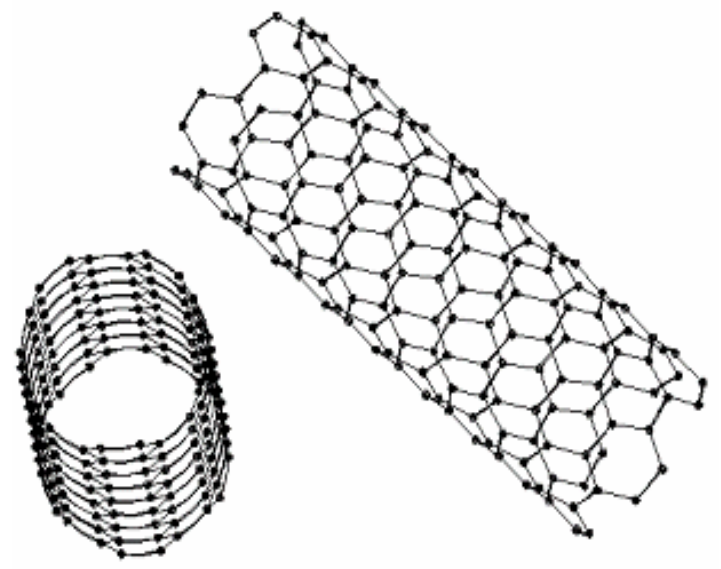

Fig. 1. An armchair polyhex nanotube $\operatorname{TUVC}_{6}[17,10]$

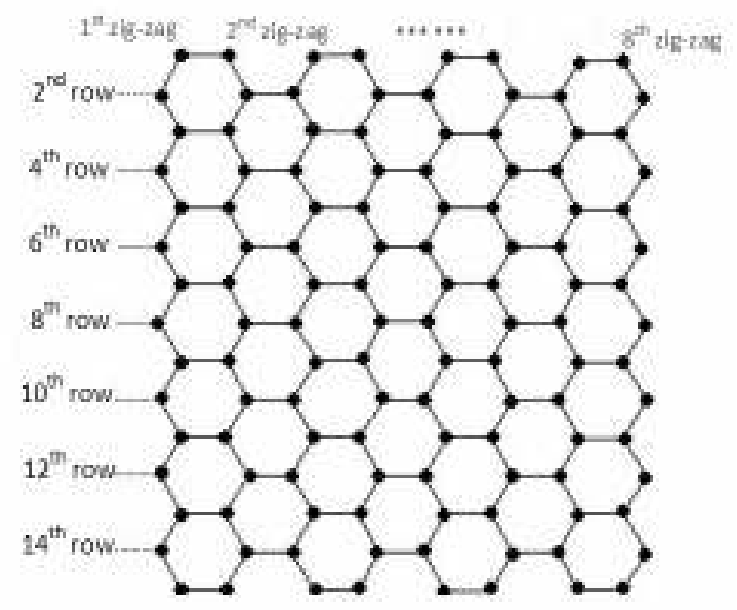

Fig. 2. An armchair polyhex lattice with $p=15$ and $q=8$

Theorem 1. Suppose that $p$ is odd. Then

$$
\xi(G)= \begin{cases}q^{2}(3 p-2) & p \leq \frac{q}{2}+1 \\ \frac{3}{4} q^{3}+\frac{1}{2} q^{2}+2 q-5 q p+3 q p^{2} & \frac{q}{2}+1<p \leq q-1 \\ \frac{9}{4} q p^{2}-\frac{7}{2} q p+\frac{3}{2} q^{2} p-q^{2}+\frac{5}{4} q & p \geq q+1\end{cases}
$$

Proof. We first notice that if $x$ and $y$ are vertices in the same row of nanotube then $\delta(x)=\varepsilon(y)$. Consider three cases that (i) $p \leq q / 2+1$; (ii) $q / 2+1<p \leq q-1$; and, (iii) $p \geq q+1$. Suppose $p \leq q / 2+1$. From Figure $3 \mathrm{a}$, one can see that for every vertex $x, \varepsilon(x)=q$. If $p \geq q+1$ then $(p+q-$ $1) / 2 \leq \varepsilon(x) \leq p+q / 2-1$. In this case, if $x$ is a vertex of $(p+1) / 2^{\text {th }}$ row of $T$ then $\varepsilon(x)=(p+q-1) / 2$. Also, by choosing a vertex y from the $i^{\text {th }}$ or $(p-i)^{\text {th }}$ row of $T$, one can see that $\varepsilon(y)=p+q / 2-i$. Finally, we assume that $q / 2+1<p \leq q-1$. Then $q \leq$ $\varepsilon(x) \leq p+q / 2-1$. From Figure $3 \mathrm{~b}$, there are $(q-p$ +2 ) rows with eccentricity $q$ and for each $i, 1 \leq i \leq$ $p-q / 2-1$, there are two rows with $\varepsilon=p+q / 2-i$. Apply these calculations to prove the following equations:

(i) $p \leq q / 2+1$

$$
\xi(G)=2[2 q^{2}+\underbrace{3 q^{2}+\ldots+3 q^{2}}_{(p-3) / 2}]+3 q^{2}=q^{2}(3 p-2)
$$

(ii) $\frac{q}{2}+1<p \leq q-1$

$$
\begin{aligned}
\xi(G)= & 3 q^{2}(q-p+2)+2[(2 q(p+q / 2-1)) \\
& +(3 q(p+q / 2-1-1))+\ldots \\
& +(3 q(p-q / 2-1-(p-q / 2-1-1)))] \\
= & \frac{3}{4} q^{3}+\frac{1}{2} q^{2}+2 q+5 q p+3 q p^{2}
\end{aligned}
$$

(iii) $p \geq q+1$

$$
\begin{aligned}
& \xi(G)=2\left[2 q\left(p+\frac{q}{2}-1\right)+3 q\left(p+\frac{q}{2}-1-1\right)\right. \\
& +\ldots+3 q(p+q / 2-1-(p-1) / 2-1)]+ \\
& \quad+3 q\left(p+\frac{q}{2}-1-\frac{p-1}{2}\right) \\
& =\frac{18}{8} q p^{2}-\frac{14}{4} q p+\frac{3}{2} q^{2} p-q^{2}+\frac{5}{4} q
\end{aligned}
$$

This completes our proof.

In Table 1 we consider the case that $q$ is even and the eccentricities of vertices (one vertex from each row) in some nanotubes with small parameters of $p$ and $q$ are computed. In Table 2, for some nanotubes with small parameters $p$ and $q$, the number of rows with eccentricity $q$ is given. Our calculations given in the proof of Theorem 1, result from these tables. 
Table 1

The eccentricity of $T[p, q]$ ( $p$ is odd) for some vlues of $p$ and $q$

\begin{tabular}{|c|c|}
\hline The type of tubes & The sequence of eccentricities \\
\hline TUVC $_{6}[3,6]$ & $6,6,6$ \\
\hline $\mathrm{TUVC}_{6}[5,6]$ & $7,6,6,6,7$ \\
\hline $\mathrm{TUVC}_{6}[7,6]$ & $9,8,7,6,7,8,9$ \\
\hline $\mathrm{TUVC}_{6}[9,6]$ & $11,10,9,8,7,8,9,10,11$ \\
\hline $\operatorname{TUVC}_{6}[11,6]$ & $13,12,11,10,9,8,9,10,11,12,13$ \\
\hline $\operatorname{TUVC}_{6}[13,6]$ & $15,14,13,12,11,10,9,10,11,12,13,14,15$ \\
\hline $\operatorname{TUVC}_{6}[15,6] \quad 1^{\prime}$ & $17,16,15,14,13,12,11,10,11,12,13,14,15,16,17$ \\
\hline $\operatorname{TUVC}_{6}[3,8]$ & $8,8,8$ \\
\hline TUVC $_{6}[5,8]$ & $8,8,8,8,8$ \\
\hline TUVC $_{6}[7,8]$ & $10,9,8,8,8,9,10$ \\
\hline TUVC $_{6}[9,8]$ & $12,11,10,9,8,9,10,11,12$ \\
\hline $\operatorname{TUVC}_{6}[11,8]$ & $14,13,12,11,10,9,10,11,12,13,14$ \\
\hline $\operatorname{TUVC}_{6}[13,8]$ & $16,15,14,13,12,11,10,11,12,13,14,15,16$ \\
\hline $\operatorname{TUVC}_{6}[15,8] \quad 18$ & $18,17,16,15,14,13,12,11,12,13,14,15,16,17,18$ \\
\hline $\operatorname{TUVC}_{6}[3,10]$ & $10,10,10$ \\
\hline TUVC $_{6}[5,10]$ & $10,10,10,10,10$ \\
\hline TUVC $_{6}[7,10]$ & $11,10,10,10,10,10,11$ \\
\hline TUVC $_{6}[9,10]$ & $13,12,11,10,10,10,11,12,13$ \\
\hline $\mathrm{TUVC}_{6}[11,10]$ & $15,14,13,12,11,10,11,12,13,14,15$ \\
\hline TUVC $_{6}[13,10]$ & $17,16,15,14,13,12,11,12,13,14,15,16,17$ \\
\hline \multicolumn{2}{|c|}{ TUVC $_{6}[15,10] 19,18,17,16,15,14,13,12,13,14,15,16,17,18,19$} \\
\hline $\operatorname{TUVC}_{6}[3,12]$ & $12,12,12$ \\
\hline TUVC $_{6}[5,12]$ & $12,12,12,12,12$ \\
\hline $\operatorname{TUVC}_{6}[7,12]$ & $11,10,10,10,10,10,11$ \\
\hline $\mathrm{TUVC}_{6}[9,12]$ & $14,13,12,12,12,12,12,13,14$ \\
\hline TUVC $_{6}[11,12]$ & $16,15,14,13,12,12,12,13,14,15,16$ \\
\hline $\mathrm{TUVC}_{6}[13,12]$ & $18,17,16,15,14,13,12,13,14,15,16,17,18$ \\
\hline \multicolumn{2}{|c|}{ TUVC $_{6}[15,12] 20,19,18,17,16,15,14,13,14,15,16,17,18,19,20$} \\
\hline $\operatorname{TUVC}_{6}[3,14]$ & $14,14,14$ \\
\hline $\operatorname{TUVC}_{6}[5,14]$ & $14,14,14,14,14$ \\
\hline $\operatorname{TUVC}_{6}[7,14]$ & $14,14,14,14,14,14,14$ \\
\hline $\operatorname{TUVC}_{6}[9,14]$ & $15,14,14,14,14,14,14,14,15$ \\
\hline TUVC $_{6}[11,14]$ & $17,16,15,14,14,14,14,14,15,16,17$ \\
\hline $\mathrm{TUVC}_{6}[13,14]$ & $19,18,17,16,15,14,13,14,15,16,17,18,19$ \\
\hline \multicolumn{2}{|c|}{$\operatorname{TUVC}_{6}[15,14] 21,20,19,18,17,16,15,14,15,16,17,18,19,20,21$} \\
\hline $\operatorname{TUVC}_{6}[3,16]$ & $16,16,16$ \\
\hline $\operatorname{TUVC}_{6}[5,16]$ & $16,16,16,16,16$ \\
\hline $\operatorname{TUVC}_{6}[7,16]$ & $16,16,16,16,16,16,16$ \\
\hline $\operatorname{TUVC}_{6}[9,16]$ & $16,16,16,16,16,16,16,16,16$ \\
\hline $\mathrm{TUVC}_{6}[11,16]$ & $18,17,16,16,16,16,16,16,16,17,18$ \\
\hline TUVC $_{6}[13,16]$ & $20,19,18,17,16,16,16,16,16,17,18,19,20$ \\
\hline $\operatorname{TUVC}_{6}[15,16] 22$ & $22,21,20,19,18,17,16,16,16,17,18,19,20,21,22$ \\
\hline
\end{tabular}

Table 2

The number of rows with eccentricity $q$, when $p$ is odd

\begin{tabular}{llllllllllll}
\hline \hline$p$ & 3 & 5 & 7 & 9 & 11 & 13 & 15 & 17 & 19 & 21 & 23 \\
\hline 6 & 3 & 3 & 0 & 0 & 0 & 0 & 0 & 0 & 0 & 0 & 0 \\
8 & 3 & 5 & 3 & 0 & 0 & 0 & 0 & 0 & 0 & 0 & 0 \\
10 & 3 & 5 & 5 & 3 & 0 & 0 & 0 & 0 & 0 & 0 & 0 \\
12 & 3 & 5 & 7 & 5 & 3 & 0 & 0 & 0 & 0 & 0 & 0 \\
14 & 3 & 5 & 7 & 7 & 5 & 3 & 0 & 0 & 0 & 0 & 0 \\
16 & 3 & 5 & 7 & 9 & 7 & 5 & 3 & 0 & 0 & 0 & 0 \\
18 & 3 & 5 & 7 & 9 & 9 & 7 & 5 & 3 & 0 & 0 & 0 \\
20 & 3 & 5 & 7 & 9 & 11 & 9 & 7 & 5 & 3 & 0 & 0 \\
22 & 3 & 5 & 7 & 9 & 11 & 11 & 9 & 7 & 5 & 3 & 0 \\
24 & 3 & 5 & 7 & 9 & 11 & 13 & 11 & 9 & 7 & 5 & 3 \\
\hline \hline
\end{tabular}

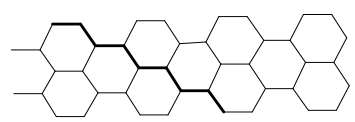

(a)

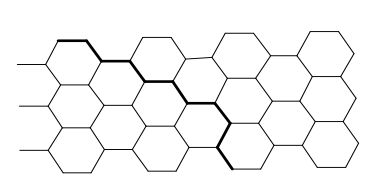

(b)

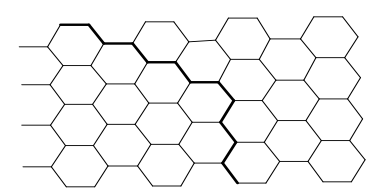

(c)
Fig. 3. The longest path containing a vertex at the first row, when $p$ is odd and (a) $p \leq q / 2+1$; (b) $q / 2+1<p \leq q-1$; (c) $p \geq q+1$

Theorem 2. Suppose that $p$ is even. Then

$$
\xi(G)= \begin{cases}q^{2}(3 p-2) & p \leq \frac{q}{2}+1 \\ \frac{3}{4} q^{3}+\frac{1}{2} q^{2}+2 q-5 q p+3 q p^{2} & \frac{q}{2}+1<q+2 \\ \frac{9}{4} q p^{2}-\frac{7}{2} q p+\frac{3}{2} q^{2} p-q^{2}+\frac{5}{4} q & p \geq q+2\end{cases}
$$

Proof. We notice again that if $x$ and $y$ are vertices in the same row of the nanotube then $\varepsilon(x)=$ $\varepsilon(y)$. In the same way as Theorem 1 , we consider three separate cases that (i) $p \leq q / 2+1$; (ii) $q / 2+1$ $<p<q+2$; and, (iii) $p \geq q+2$. From Figures $4 \mathrm{a}-\mathrm{c}$ and a similar method as Theorem 1, we have, 
(i) $p \leq q / 2+1$

$$
\xi(G)=2[2 q^{2}+\underbrace{3 q^{2}+\ldots+3 q^{2}}_{p / 2}]=q^{2}(3 p-2)
$$

(ii) $\frac{q}{2}+1<p<q+2$

$$
\begin{aligned}
\xi(G)= & 3 q^{2}(q-p+2)+2[2 q(p+q / 2-1) \\
& +3 q(p+q / 2-1-1) \\
& +\ldots+3 q(p+q / 2-1(p-q / 2-1-1))] \\
= & \frac{3}{4} q^{3}+\frac{1}{2} q^{2}+2 q-5 q p+3 q p^{2}
\end{aligned}
$$

(iii) $p \geq q+2$

$$
\begin{aligned}
\xi(G) & =2[q(p+q / 2-1)+3 q(p+q / 2-1-1) \\
& +3 q(p+q / 2-1-2)+\ldots \\
& +3 q(p+q / 2-1-(p / 2-1))] \\
& =-q^{2}-\frac{7}{2} q p+2 q+\frac{9}{4} q p^{2}+\frac{3}{2} q^{2} p
\end{aligned}
$$

This completes our argument.

In Table 3 we consider the case that $q$ is odd and the eccentricities of vertices (one vertex from each row) in some nanotubes with small parameters of $p$ and $q$ are computed. In Table 4 we compute the number of rows with eccentricity $q$, for some nanotubes with small parameters $p$ and $\mathrm{q}$. Our calculations given in the proof of Theorem 2, result from these tables.

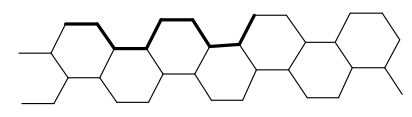

(a)

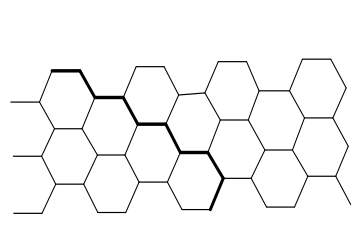

(b)

\begin{tabular}{|c|c|}
\hline $\begin{array}{l}\text { The type of } \\
\text { tubes }\end{array}$ & The sequence of eccentricities \\
\hline $\operatorname{TUVC}_{6}[4,4]$ & $5,4,4,5$ \\
\hline $\mathrm{TUVC}_{6}[6,4]$ & $7,6,5,5,6,7$ \\
\hline $\operatorname{TUVC}_{6}[8,4]$ & $9,8,7,6,6,7,8,9$ \\
\hline $\operatorname{TUVC}_{6}[10,4]$ & $11,10,9,8,7,7,8,9,10,11$ \\
\hline $\operatorname{TUVC}_{6}[12,4]$ & $13,12,11,10,9,8,8,9,10,11,12,13$ \\
\hline $\operatorname{TUVC}_{6}[14,4]$ & $15,14,13,12,11,10,9,9,10,11,12,13,14,15$ \\
\hline \multicolumn{2}{|l|}{$\operatorname{TUVC}_{6}[16,4] 1$} \\
\hline $\operatorname{TUVC}_{6}[4,6]$ & $6,6,6,6$ \\
\hline $\operatorname{TUVC}_{6}[6,6]$ & $8,7,6,6,7,8$ \\
\hline $\mathrm{TUVC}_{6}[8,6]$ & $10,9,8,7,7,8,9,10$ \\
\hline $\operatorname{TUVC}_{6}[10,6]$ & $12,11,10,9,8,8,9,10,11,12$ \\
\hline $\operatorname{TUVC}_{6}[12,6]$ & $14,13,12,11,10,9,9,10,11,12,13,14$ \\
\hline TUVC $_{6}[14,6]$ & $16,15,14,13,12,11,10,10,11,12,13,14,15,16$ \\
\hline \multicolumn{2}{|l|}{$\operatorname{TUVC}_{6}[16,6] 1$} \\
\hline $\operatorname{TUVC}_{6}[4,8]$ & $8,8,8,8$ \\
\hline $\operatorname{TUVC}_{6}[6,8]$ & $9,8,8,8,8,9$ \\
\hline $\mathrm{TUVC}_{6}[8,8]$ & $11,10,9,8,8,9,10,11$ \\
\hline $\operatorname{TUVC}_{6}[10,8]$ & $13,12,11,10,9,9,10,11,12,13$ \\
\hline $\operatorname{TUVC}_{6}[12,8]$ & $15,14,13,12,11,10,10,11,12,13,14,15$ \\
\hline $\operatorname{TUVC}_{6}[14,8]$ & $17,16,15,14,13,12,11,11,12,13,14,15,16,17$ \\
\hline $\operatorname{TUVC}_{6}[16,8]$ & $19,18,17,16,15,14,13,12,12,13,14,15,16,17,18,19$ \\
\hline $\operatorname{TUVC}_{6}[4,10]$ & $10,10,10,10$ \\
\hline $\operatorname{TUVC}_{6}[6,10]$ & $10,10,10,10,10,10$ \\
\hline $\operatorname{TUVC}_{6}[8,10]$ & $12,11,10,10,10,10,11,12$ \\
\hline TUVC6[10,10] & $14,13,12,11,10,10,11,12,13,14$ \\
\hline $\mathrm{TUVC}_{6}[12,10]$ & $16,15,14,13,12,11,11,12,13,14,15,16$ \\
\hline $\operatorname{TUVC}_{6}[14,10]$ & $18,17,16,15,14,13,12,12,13,14,15,16,17,18$ \\
\hline \multicolumn{2}{|l|}{$\operatorname{TUVC}_{6}[16,10] 20}$, \\
\hline $\operatorname{TUVC}_{6}[4,12]$ & $12,12,12,12$ \\
\hline $\operatorname{TUVC}_{6}[6,12]$ & $12,12,12,12,12,12$ \\
\hline $\operatorname{TUVC}_{6}[8,12]$ & $13,12,12,12,12,12,12,13$ \\
\hline $\operatorname{TUVC}_{6}[10,12]$ & $15,14,13,12,12,12,12,13,14,15$ \\
\hline $\mathrm{TUVC}_{6}[12,12]$ & $17,16,15,14,13,12,12,13,14,15,16,17$ \\
\hline $\operatorname{TUVC}_{6}[14,12]$ & $19,18,17,16,15,14,13,13,14,15,16,17,18,19$ \\
\hline \multicolumn{2}{|l|}{$\operatorname{TUVC}_{6}[16,12] 21$} \\
\hline $\operatorname{TUVC}_{6}[4,14]$ & $14,14,14,14$ \\
\hline $\operatorname{TUVC}_{6}[6,14]$ & $14,14,14,14,14,14$ \\
\hline $\operatorname{TUVC}_{6}[8,14]$ & $14,14,14,14,14,14,14,14$ \\
\hline TUVC $_{6}[10,14]$ & $16,15,14,14,14,14,14,14,14,15,16$ \\
\hline TUVC $_{6}[12,14]$ & $18,17,16,15,14,14,14,14,15,16,17,18$ \\
\hline TUVC $_{6}[14,14]$ & $20,19,18,17,16,15,14,14,15,16,17,18,19,20$ \\
\hline TUVC $_{6}[16,14] 2$ & $22,21,20,19,18,17,16,15,15,16,17,18,19,20,21,22$ \\
\hline
\end{tabular}

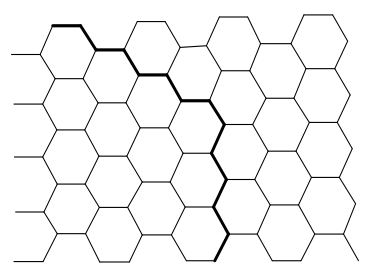

(c)
Fig. 4. The longest path containing a vertex at the first row, when $p$ is even and (a) $p \leq q / 2+1$; (b) $q / 2+1<p<q+2$; (c) $p \geq q+2$
Table 3

The Eccentricity of $T[p, q]$ ( $p$ is even), for some values of $p$ and $q$. 
Table 4

The number of rows with eccentricity of $q$, when $p$ is even

\begin{tabular}{|c|c|c|c|c|c|c|c|c|c|c|}
\hline & 4 & 6 & 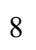 & 10 & 12 & 14 & 16 & 18 & 20 & 22 \\
\hline & 2 & 0 & 0 & 0 & 0 & 0 & 0 & 0 & 0 & 0 \\
\hline 6 & 4 & 2 & 0 & 0 & 0 & 0 & 0 & 0 & 0 & 0 \\
\hline 0 & 4 & 4 & 2 & 0 & 0 & 0 & 0 & 0 & 0 & 0 \\
\hline 10 & 4 & 6 & 4 & 2 & 0 & 0 & 0 & 0 & 0 & 0 \\
\hline 12 & 4 & 6 & 6 & 4 & 2 & 0 & 0 & 0 & 0 & 0 \\
\hline 14 & 4 & 6 & 8 & 6 & 4 & 2 & 0 & 0 & 0 & 0 \\
\hline 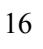 & 4 & 6 & 8 & 0 & 6 & 4 & 2 & 0 & 0 & 0 \\
\hline 18 & 4 & 6 & 8 & 10 & 8 & 6 & 4 & 2 & 0 & 0 \\
\hline 20 & 4 & 6 & 8 & 10 & 10 & 8 & 6 & 4 & 2 & 0 \\
\hline 22 & 4 & 6 & 8 & 10 & 12 & 10 & 8 & 6 & 4 & 2 \\
\hline
\end{tabular}

We can combine Theorems 1 and 2 in the following theorem:

Theorem. Suppose $T=T[p, q]$ denotes an armchair polyhex nanotube with parameters $p$ and $q$. Then the eccentric connectivity index of $T$ is computed as follows:

$$
\xi(G)= \begin{cases}q^{2}(3 p-2) & p \leq \frac{q}{2}+1 \\ \frac{3}{4} q^{3}+\frac{1}{2} q^{2}+2 q-5 q p+3 q p^{2} & \frac{q}{2}+1<p \leq q \\ \frac{9}{4} q p^{2}-\frac{7}{2} q p+\frac{3}{2} q^{2} p-q^{2}+\frac{5}{4} q & p \geq q+1\end{cases}
$$

\section{CONCLUSIONS}

In this paper the eccentric connectivity index of an armchair nanotube is computed for the first time. To the best of our knowledge it is the first paper considering the eccentric connectivity index of nanotubes into account. We present a powerful method for calculating such indices. It is possible to extend our method to a zig-zag polyhex nanotube.

\section{REFERENCES}

[1] V. Sharma, R. Goswami and A. K. Madan, Eccentric connectivity index: A novel highly discriminating topological descriptor for structure-property and structure-activity studies, J. Chem. Inf. Comput. Sci., 37, 273-282 (1997).

[2] H. Dureja and A. K. Madan, Superaugmented eccentric connectivity indices: new-generation highly discriminating topological descriptors for QSAR/QSPR modeling, Med. Chem. Res., 16, 331-341 (2007).

[3] V. Kumar, S. Sardana and A. K. Madan, Predicting antiHIV activity of 2,3-diary 1-1,3-thiazolidin-4-ones: computational approaches using reformed eccentric connectivity index, J. Mol. Model, 10, 399-407 (2004).

[4] S. Sardana and A. K. Madan, Application of graph theory: Relationship of molecular connectivity index, Wiener's index and eccentric connectivity index with diuretic activity, MATCH Commun. Math. Comput. Chem., 43, 8598 (2001).

[5] M. Fischermann, A. Homann, D. Rautenbach, L. A. Szekely and L. Volkmann, Wiener Index versus maximum degree in trees, Discrete Appl. Math., 122, 127-137 (2002).

[6] S. Gupta, M. Singh and A. K. Madan, Application of Graph Theory: Relationship of Eccentric Connectivity Index and Wiener's Index with Anti-inflammatory Activity, J. Math. Anal. Appl., 266, 259-268 (2002).

[7] A. Ilić and I. Gutman, Eccentric connectivity index of chemical trees, MATCH Commun. Math. Comput. Chem., to appear.

[8] B. Zhou and Z. Du, On eccentric connectivity index, MATCH Commun. Math. Comput. Chem., 63, 181-198 (2010).

[9] T. Doslic, M. Saheli and D. Vukičević, Eccentric connectivity index: extremal graphs and values, submitted.

[10] A. R. Ashrafi and A. Loghman, PI index of armchair polyhex nanotubes, ARS Combinatoria, 80, 193-199 (2006).

[11] A. Iranmanesh and A. R. Ashrafi, Balaban index of an armchair polyhex, $\mathrm{TUC}_{4} \mathrm{C}_{8}(\mathrm{R})$ and $\mathrm{TUC}_{4} \mathrm{C}_{8}(\mathrm{~S})$ nanotorus, J. Comput. Theor. Nanosci., 4 (3), 514-517 (2007).

[12] H. Shabani and A. R. Ashrafi, Applications of the matrix package MATLAB in computing the wiener polynomial of armchair polyhex nanotubes and nanotori, J. Comput. Theor. Nanosci., in press.

[13] S. Yousefi and A. R. Ashrafi, Distance Matrix and Wiener Index of Armchair Polyhex Nanotubes, Studia Univ. Babes-Bolyai, Chemia, 53 (4), 111-116 (2008).

[14] H. Deng, The PI Index of TUVC6[2p; q], MATCH Commun. Math. Comput. Chem., 55, 461-476 (2006).

[15] M. Eliasi and B. Taeri, Distance in Armchair Polyhex Nanotubes, MATCH Commun. Math. Comput. Chem. 62 295-310 (2009).

[16] M. V. Diudea, M. Stefu, B. Pârv and P. E. John, Armchair Polyhex Nanotubes, Croat. Chem. Acta, 77 (1-2), 111-115 (2004).

[17] F. Harary, Graph Theory, Addison-Wesley, Reading MA, 1969. 